\title{
Artificial Intelligence for Estimating Laser Power from Temperature Distribution
}

\author{
Miki Nakaone ${ }^{1}$, Tomomasa Ohkubo*1, Yuki Ueno ${ }^{1}$, Ken Goto ${ }^{2}$, and Yutaka Kagawa ${ }^{3}$ \\ ${ }^{1}$ Graduate School of Engineering, Tokyo University of Technology, Japan \\ 2 Japan Aerospace Exploration Agency, Japan \\ ${ }^{3}$ Katayanagi Institute, Tokyo University of Technology, Japan \\ ${ }^{*}$ Corresponding author's e-mail: ookubotmms@stf.teu.ac.jp
}

\begin{abstract}
We developed a laser heating test system called the selective laser thermoregulation (SLT) system using a fiber laser and a galvano mirror system to confirm the reliability of SiC/SiC CMCs, which are expected to improve the efficiency of aircraft engines. However, to realize the required temperature distribution using the SLT system, many parameters must be determined. For automatic determination of the parameters, we developed AI for estimating the laser power needed to reproduce the required temperature distribution. We created datasets for machine learning by numerical simulation of laser heating and compared three types of fully connected neural networks. The AI learned the relationship between laser power and the temperature distribution and then estimated the laser power from an untrained temperature distribution. The $R^{2}$ of the 2-layer neural network was 1.64 , while the $R^{2}$ values of the 3- and 4-layer neural networks were 0.98 and 0.99 , respectively. Thus, we found that three or four layers of fully connected neural network are sufficient for realizing AI which can estimate laser power from a temperature distribution.
\end{abstract}

DOI: 10.2961/jlmn.2021.02.2002

Keywords: selective laser thermoregulation (SLT) method, deep learning, ceramic matrix composites (CMCs), galvano scanner, fiber laser

\section{Introduction}

Increasing demands are being placed on aircraft in recent decades, because aircraft are essential for the global society.

$\mathrm{SiC} / \mathrm{SiC}$ ceramic composites (CMCs) are expected to replace Ni-based alloys as new materials for improving the efficiency of aircraft engines [1][2]. SiC/SiC CMCs have lower density and higher heat resistance than the present alloys and are promising materials for aircraft engines. However, their reliability should be confirmed by heating tests.

Many studies have measured the physical properties of SiC/SiC CMCs in a high-temperature environment [3]-[6]. However, in actual use in an engine, SiC/SiC CMCs will be heated and cooled repeatedly for a long period of time. Therefore, accelerated heating tests that repeat heating and cooling over an extended period are necessary to confirm their reliability.

A heating test method using a $\mathrm{CO}_{2}$ laser has been developed to shorten the test time [7]. It is possible to heat locally and to repeatedly heat and cool rapidly by using a laser. However, water vapor is generated inside aircraft engines and it absorbs $\mathrm{CO}_{2}$ laser light. Therefore, it is hard to test CMCs in an environment simulating the inside of an aircraft engine.

Therefore, a heating test system using a fiber laser was recently reported by Whitlow et al [8]. The wavelength of the fiber laser is $1.07 \mu \mathrm{m}$ and its absorption coefficient for water vapor is much smaller than that of the $\mathrm{CO}_{2}$ laser [9].

In previous laser heating test studies, the laser beam is shaped into a rectangle by static optics before the laser is irradiated on the test piece to heat a rectangular area. However, with this method, the outer edge of the irradiated surface tends to be cooled. In addition, if the cooling conditions change due to changes in the gas flow around the test piece, it is difficult for static optics to adjust the input power distribution dynamically. Therefore, it is necessary to heat the surface uniformly and control the input power distribution dynamically.

We developed a heating test system called the selective laser thermoregulation (SLT) system. The SLT system can dynamically adjust the input power distribution using a fiber laser and a galvano mirror system if there are any changes in the cooling conditions or shape of a target [10].

However, the SLT system requires the user to determine many parameters such as the laser power, the spot size, and the scanning path to realize the required temperature distribution. Moreover, if the required temperature distribution, the shape of the test piece, or the cooling conditions change, many parameters will need to be determined again according to the changes. To accelerate research on the laser heating test, AI to estimate the irradiation conditions to achieve the required temperature distribution is necessary.

In this study, we propose the development of AI for estimating laser power to reproduce a required temperature distribution. In typical laser heating studies, we determine the laser power first, then the temperature distribution is obtained as the result. On the other hand, in this study, the required temperature distribution is input to the developed $\mathrm{AI}$, and then the $\mathrm{AI}$ estimates the laser power to realize the temperature distribution. It is easy to determine a number 
of parameters with AI for several required temperature distributions and test piece shapes.

\section{Research method}

Our research consisted of three steps. Firstly, we created an input dataset of temperature distributions by numerical simulation of laser heating. The input for the simulation was the irradiation conditions of the laser and the properties of the heating target. The output of the simulation was the temperature distribution. Secondly, the AI learned the relationship between the temperature distribution and the laser power. Finally, the laser power could be estimated by the AI even from an untrained temperature distribution.

\subsection{Input datasets from numerical simulation}

We created datasets for learning and estimation by numerical simulation of laser heating. The governing equations shown in equation (1) were calculated using the finite difference method in two dimensions, considering the laser input power, thermal conduction of the target material, and thermal loss to the air due to heat transfer and radiation.

$$
\rho V C \frac{\partial T}{\partial t}=\alpha L A+C(T)-H(T)-R(T)
$$

$L, C(T), H(T)$, and $R(T)$ are the laser input power [W], thermal conduction [W], heat transfer [W], and radiation [W], respectively. $\rho, V, C, T, t, \alpha$, and $A$ are the density $\left[\mathrm{kg} / \mathrm{m}^{3}\right]$, volume $\left[\mathrm{m}^{3}\right]$, specific heat $[\mathrm{J} /(\mathrm{kg} \cdot \mathrm{K})]$, temperature $[\mathrm{K}]$, time $[\mathrm{s}]$, absorption rate, and area $\left[\mathrm{m}^{2}\right]$, respectively. The details of $L, C(T), H(T)$, and $R(T)$ in equation (1) are shown in equation (2) to equation (5).

$$
\begin{aligned}
& L=P(1-\gamma) \\
& C(T)=\nabla(\lambda \nabla T) V \\
& H(T)=h A\left(T-T_{0}\right) \\
& R(T)=\varepsilon \sigma A\left(T^{4}-T_{0}^{4}\right)
\end{aligned}
$$

$P$ and $\gamma$ used in equation (2) are the laser power [W] and the reflectivity of the laser at the target surface, respectively. $\lambda$ used in equation (3) is the thermal conductivity $[\mathrm{W} /(\mathrm{m} \cdot \mathrm{K})] \cdot h$ and $T_{0}$ in equation (4) are the heat transfer coefficient and the background temperature [K], respectively. $\varepsilon$ and $\sigma$ in equation (5) are the emissivity and the Stefan-Boltzmann constant, respectively.

As shown in Fig. 1, the boundary conditions are a heat transfer boundary condition of $h=5.0 \mathrm{~W} / \mathrm{m}^{2} \cdot \mathrm{K}$ for the area in contact with the test piece gripping area, and $h=10.0 \mathrm{~W} / \mathrm{m}^{2} \cdot \mathrm{K}$ for the other boundary areas.

We used the Cartesian coordinate system, and the numbers of grid points in the $x$ and $y$ directions were 41 and 9 respectively. The time step was $1.0 \times 10^{-3} \mathrm{~s}$.

The shape of the test piece was $40 \mathrm{~mm} \times 8 \mathrm{~mm} \times 2 \mathrm{~mm}$ (thickness). The coordinate system had the origin at the center of the test piece. The laser with a radius of $4 \mathrm{~mm}$ was irradiated on a single point.

An example of the numerical simulation for irradiating the laser at $x=0$ and $y=0$ is shown in Fig. 2. Numerical simulation was performed for a total of 153 different conditions: 9 different laser power values ranging from $400 \mathrm{~W}$ to $4000 \mathrm{~W}$ in $450 \mathrm{~W}$ increments, and 17 different irradiation positions values ranging from $-16 \mathrm{~mm}$ to $16 \mathrm{~mm}$ in 2 mm increments in the $x$ direction. All the data generated were the calculated results for 10 seconds after laser irradiation.

We divided the data into training data and test data, randomly dividing the 153 datasets into $80 \%$ training data and $20 \%$ test data. Training data were used for learning, while test data were used to confirm the generality of the developed AI, such as whether the AI overfit the training data.

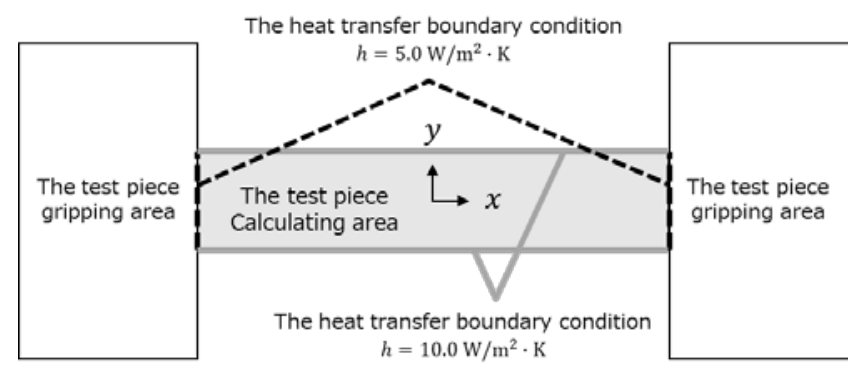

Fig. 1 The boundary conditions. The grayed area is the calculated area.

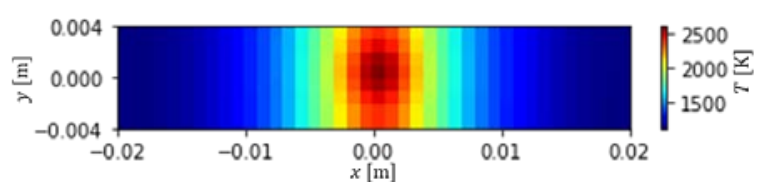

Fig. 2 Visualizing the calculated temperature distribution when the laser is irradiated at $\mathrm{x}=0 \mathrm{~mm}, \mathrm{y}=0 \mathrm{~mm}$ by numerical calculation.

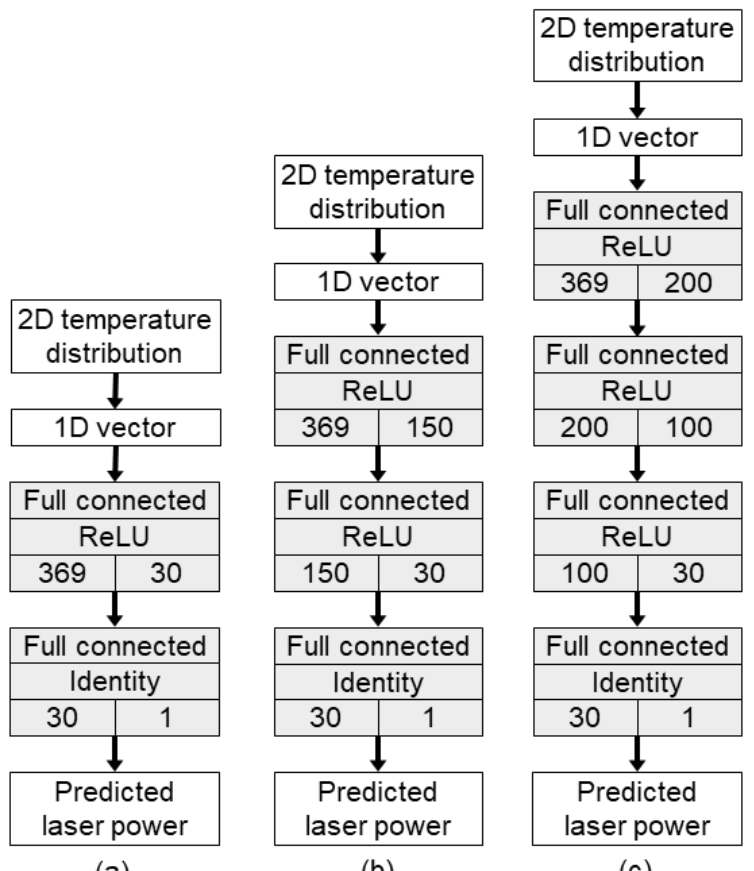

(a)

(b)

(c)

Fig. 3 Neural network configuration for estimating laser power from temperature distribution.

(a) 2 layers, (b) 3 layers, (c) 4 layers 


\begin{tabular}{|c|c|}
\hline \multicolumn{2}{|c|}{ Layer } \\
\hline \multicolumn{2}{|c|}{ Activation function } \\
\hline $\begin{array}{c}\text { Input } \\
\text { nodes }\end{array}$ & $\begin{array}{c}\text { Output } \\
\text { nodes }\end{array}$ \\
\hline
\end{tabular}

Fig. 4 Legend of a block used in Fig. 3.

\subsection{Neural network}

In this study, we developed and compared 3 neural networks as shown in Fig. 3. The legends used in Fig. 3 are shown in Fig. 4, which describes the types of layers, the activation functions, and the number of input and output nodes used in each layer. The three neural networks had different numbers of layers and nodes. However, the following procedures were the same. Firstly, the 2D temperature distribution with 9 rows and 41 columns was flattened to a $1 \mathrm{D}$ vector of 369 elements. After the conversion of the input data, all the layers were fully connected. In the fully connected layers, all the activation functions but the last layer are ReLU functions [11] and the last is the identity function, the loss function is the mean square error, and the optimizer is root mean square propagation.

\section{Evaluation of the AI}

As mentioned in section 2, the AI learns the relationship between the irradiation conditions and temperature distribution using training data. The results are evaluated by the mean absolute error (MAE). MAE is expressed in equation (6).

$$
\mathrm{MAE}=\frac{1}{n} \sum_{k=1}^{n}\left|y_{k}-t_{k}\right|
$$

where $n, y_{k}$, and $t_{k}$ are the number of data, the $k$-th predicted value, and the $k$-th correct value, respectively.

Furthermore, the test data are used for final evaluation of whether the AI can estimate the correct laser power even from the untrained test data. The results are evaluated by $R^{2}$. As mentioned in section 2.1, 153 data were randomly divided into $80 \%$ training data and $20 \%$ test data. Therefore, we performed the following series of steps three times: dividing the dataset, learning with the training data, estimating the laser power from the untrained data using the test data, and evaluating the results by $R^{2} \cdot R^{2}$ is expressed in equation (7).

$$
R^{2}=1-\frac{\sum_{i=1}^{n}\left(y_{i}-t_{i}\right)^{2}}{\sum_{i=1}^{n}\left(y_{i}-\bar{y}\right)^{2}}
$$

where $y_{i}, t_{i}$, and $\bar{y}$ are the $i$-th predicted value, the $i$-th correct value, and the average of the predicted values, respectively.

We set two hyperparameters: epoch numbers and batch size. We set the epoch numbers to 250 and the batch size to 32. (a)

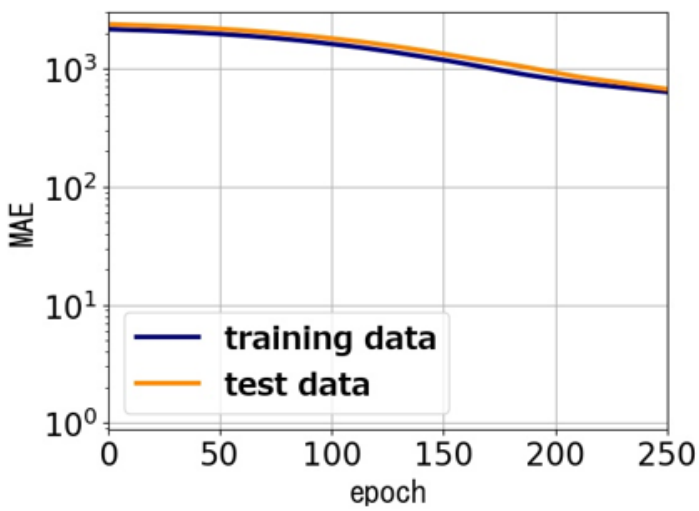

(b)

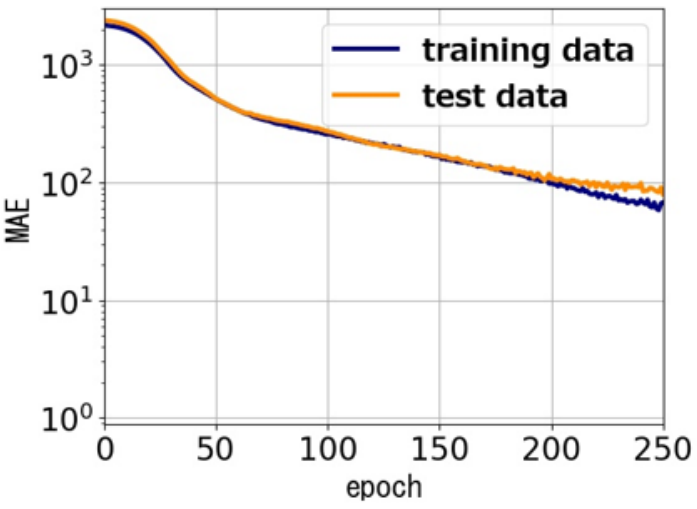

(c)

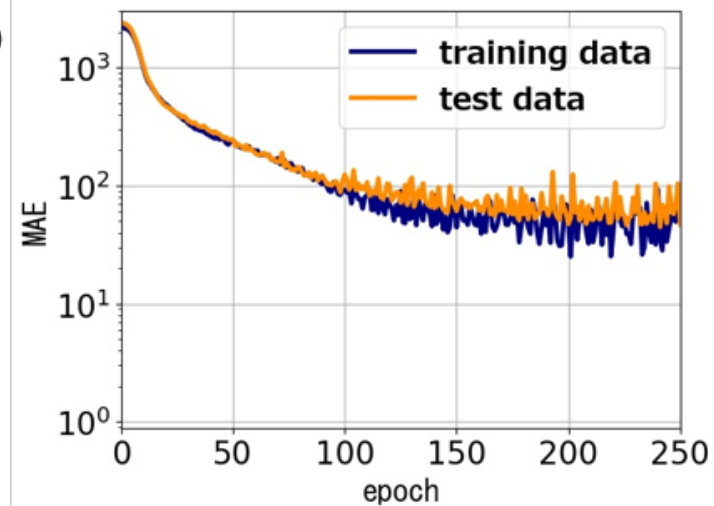

Fig. 5 MAE of each neural network during training and testing.

(a) 2 layers, (b) 3 layers, (c) 4 layers

\section{Results}

Fig. 5 shows one case of the relationship between epoch and MAE for each neural network. The blue line is the results using training data and the orange lines are the results using test data. In all cases, the blue line and the orange line follow approximately the same trajectory. In Fig. 5(c), although the training data seem to deviate from the test data when the number of epochs is more than 100, the vertical axis is a log scale and the MAE itself is sufficiently small. Therefore, the data are not overlearned.

Fig. 6 shows the relationship between the exact input power and the predicted input power using test data. The points are all the results of three trials.

Moreover, the $R^{2}$ of the 2-layer neural network is -1.64 , that of the 3-layer neural network is 0.98 , and that of the 4-layer neural network is $0.99 . R^{2}$ is the average value of the three trials. 
(a)

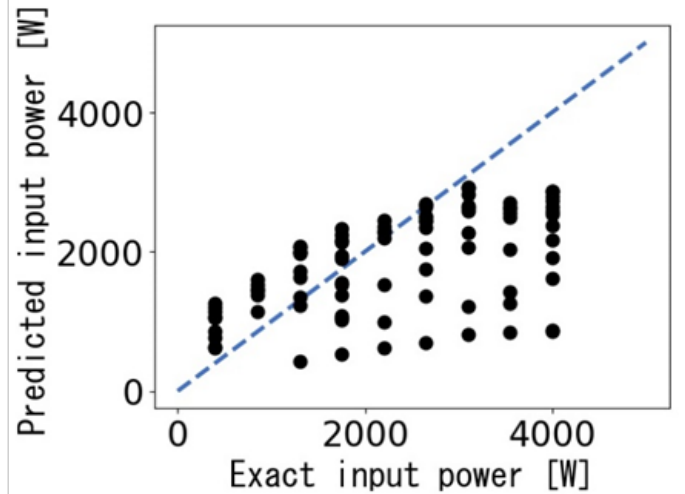

(b)

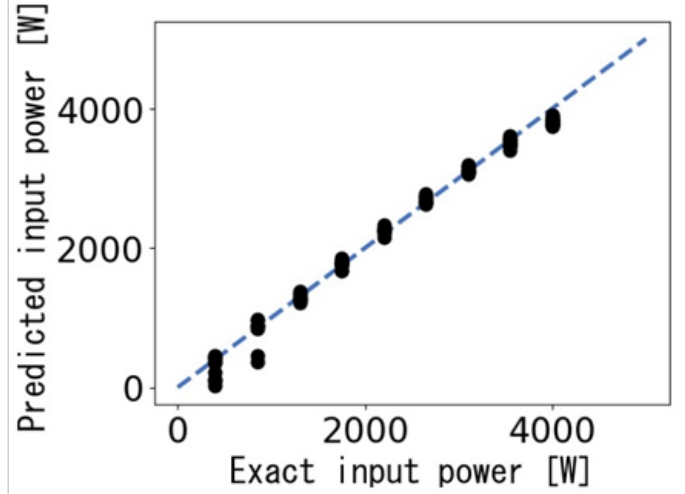

(c)

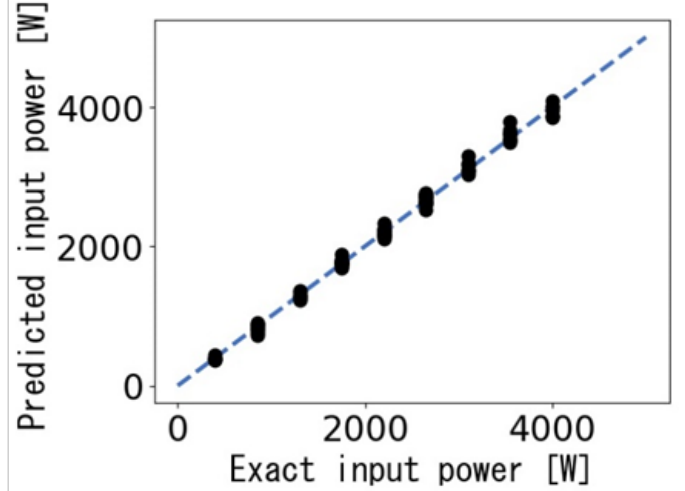

Fig. 6 The relationship between the exact input power and the predicted input power by each neural network using untrained test data.

(a) 2 layers, (b) 3 layers, (c) 4 layers

\section{Discussion}

Fig. 5 shows that MAE decreased as learning progressed in all cases. From these results, we confirmed that the parameters such as the weights and the bias inside the neural network were well optimized, which means the AI successfully learned the relationship between the laser irradiation conditions and the temperature distributions. Furthermore, there was not much difference between the MAE results of the training and test data. Therefore, we confirmed that the AI was able to learn the training data without problems such as overfitting.

Fig. 6(a) shows that the AI with the 2-layer neural network estimated the laser power differently from the exact input power because the points are not on the dashed line. Fig. 6(b) and Fig. 6(c) show that the AI with the 3-layer or 4-layer neural network gave an estimation of the laser power close to the exact input power, because the points are aligned along the dashed line. Moreover, we obtained values of 0.98 and 0.99 for $R^{2}$ from the predicted results using the 3-layer and 4-layer neural networks, which are close to 1 , although the $R^{2}$ of the 2-layer neural network was -1.64. Therefore, we found that adding layers to the neural network is effective in estimating the laser power from a temperature distribution using a fully connected neural network, but 4 layers are sufficient.

\section{Conclusion and future work}

We succeeded in developing AI for estimating laser power from a temperature distribution. The 3-layer and 4layer neural networks, which had deeper layers than the 2layer neural network, could estimate laser power more accurately. The $R^{2}$ values for the 3-layer and 4-layer neural networks were 0.98 and 0.99 , respectively.

In this study, the AI estimated only the laser power from the temperature distribution. However, the irradiation conditions of the laser actually involve more parameters. Therefore, we will increase the number of parameters to be estimated in future work.

\section{Acknowledgments and Appendixes}

This paper is based on results obtained from a project, JPNP20004, subsidized by the New Energy and Industrial Technology Development Organization (NEDO).

\section{References}

[1] G.S. Corman and K.L. Luthra: "Handbook of Ceramic Composites” ed. by N. P. Bansal, (Publisher, Boston, 2005) p.99

[2] M. Roode, J. Price, J. Kimmel, N. Miriyala, D. Leroux, A. Fahme, and K. Smith: J. Eng. Gas Turbines Power, 129, (2007) 21.

[3] R. J. Young, A. B. L. Broadbridge, and C.-L. So: J. Microscopy, 196, (1999) 257.

[4] Y. L. Dong, H. Kakisawa, and Y. Kagawa: Meas. Sci. Technol., 25, (2014) 025002.

[5] A. Haboub, H. A. Bale, J. R. Nasiatka, B. N. Cox, D.B. Marshall, R. O. Ritchie, and A. A. MacDowell: Rev. Sci. Instrum., 85, (2014) 083702.

[6] T. Whitlow, E. Jones, and C. Przybyla: Composite Structures, 158, (2016) 245.

[7] M.P. Appleby, D. Zhu, and G. Morscher: surf Coat technol, 284, (2015) 318.

[8] T. Whitlowa, J. Pitzb, J. Piercea, S. Hawkinsc, A. Samuelc, K. Kollinsd, G. jeffersond, E. Jonesd, J. Vernond, and C. Przybylad: Composite Structures, 210, (2019) 179.

[9] W. M. Irvine and J. B. Pollack, "Infrared optical properties of water and ice spheres," Icarus, 8, (1968) , 324-360.

[10]H. Koshiji, T. Ohkubo, K. Azato, Y. Kameda, E. Matsunaga, T. Dobashi, N. Shichijo, K. Goto, M. Sato, C. Fujiwara, and Y. Kagawa: J. Laser Micro/Nanoengineering., 15, (2020), 174.

[11] X. Glorot, A. Bordes, and Y. Bengio: Proc. 14th International Conference on Artifical Intelligence and Statistics, (2011), 315-323.

(Received: June 9, 2021, Accepted: August 29, 2021) 